\title{
Comparative Biochemical Profiles in Children Treated with Artemether-lumefantrine and Artesunate-amodiaquine for Acute Uncomplicated falciparum Malaria in Jos, North - Central Nigeria.
}

\author{
*Shwe DD, FMCPaed, **Abba OJ, B.Sc, MPH, **Akindigh, MT, B.Sc, \\ ***AdenijiBA,B.Sc, *Ebonyi AO, M.Sc, FWACP, ****Yunusa T, \\ FMCPath $* * * * *$ Pitman SL, FMCFM, FMCGP $* * * * * *$ Egah DZ, FMCPath, *Oguche S, \\ FMCPaed. \\ *Department of Paediatrics, Jos University Teaching Hospital, **Malarial Unit, APIN, JUTH, ***Nigeria \\ Institute of Medical Research, Yaba, Lagos, Nigeria, ****Department of Medical Microbiology, Abuja \\ University Teaching Hospital, Gwagwalada,FCT, *****Department of Family Medicine, Jos University \\ Teaching Hospital, Jos. ******Department of Medical Microbiology, Jos University Teaching Hospital/Faculty \\ of Medical Sciences, University of Jos.
}

\begin{abstract}
:
Background: Acute clinical episode of uncomplicated malaria induces several pathophysiological biochemical disturbances in its host. We examined and compared these biochemical alterations in children pre- and posttreatment with ACTs up to day 14 and how the ACTs affect these biochemical parameters during recovery.

Aim: To determine and compare the alterations in selected biochemical indices pre-and post-treatment of uncomplicated P. falciparum malaria with artemether-lumefantrine $(A L)$ and artesunate-amodiaquine $(A A)$ in under-five children up toDay 14 day.

Method: Data on 111 children aged 6 to 60 months who were enrolled into a drug therapeutic efficacy testing (DTET) of AL (20/120mg) with AA (25mg/67.5mg or 50/135mg) in the treatment of uncomplicated falciparum malaria, were analysed. Inclusion criteria were: history of fever in the last $24 \mathrm{hrs}$ and /or measured axillary temperature $\geq 37.5^{\circ} \mathrm{C}$, P. falciparum infection with parasitaemia $\geq 1000$ to $\leq 250,000$ parasites $/ \mu \mathrm{L}$, HIV seronegative status, and a written informed consent from parents/guardians. Enrolees were randomized into one of two treatment arms, and their biochemical indices (ALT, AST, Bilirubin and creatinine) were measured longitudinally on D0, D7 and D14 and analyzed.
\end{abstract}

Results:

Of649 subjects screened for parasitaemia , $282\left(43.5 \%\right.$ ) were febrile (temperature $\geq 37.5^{\circ} \mathrm{C}$ ), of which 252 (38.8\%) had P. falciparumparasitaemia. Parasite counts varied from 1000 - 200,000 asexual forms $\mu$ L. The mean age (months) of study population were $A L(38.9 \pm 16.90)$ and $A A(37.7 \pm 16.76)$ arms respectively, $(p=$ 0.72). Thirty- one (55.4\%) and $25(44.6 \%)$ were males and females in the ALstudy arm respectively while 32 (58.2\%) and 23 (41.8\%) were males and females respectively in the AA study arm, $(p=0.77)$. There were comparable elevations in mean serum levels of AST, ALT,creatinine and total Bilirubin from D0 to peaks by D7 with gradual decline to patients' normal at D14 post-treatment in AL and AA treatment arms. The difference was not significant. ACTs chemotherapy appeared to have no significant relationship to biochemical disturbances in the study subjects.

Conclusions: Uncomplicated paediatric Plasmodium falciparum malaria induces transient alterations in biochemical indices in Jos, North Central Nigeria. Our findings agreed with the literature. Therefore, malaria infection should be considered as a differential diagnosis in febrile children with alterations in selected biochemical indices.

Key words: Children, uncomplicated Plasmodium falciparum malaria, biochemical indices, ACTs.

\section{Introduction}

Changes in biochemical indices in acute and convalescent paediatric uncomplicated Plasmodium falciparum malaria has not been fully studied especially in children residing in most malaria endemic countries of the world. The usefulness of such knowledge increases clinical diagnosis and appreciation of biochemical toxicities of the antimalarials. ${ }^{1,2}$

Acute clinical episode of malaria induces several pathophysiological biochemical disturbances in its host. ${ }^{2,3,4}$ Many studies have examined the changes in the biochemical alterations in uncomplicated malaria 
infection; few however, have examined and compared these biochemical alterations in children pre- and posttreatment with ACTs up to day14 and how the ACTs affect these biochemical parameters during recovery.

To the best of our knowledge, there is paucity of data on the biochemical profiles of children pre and post treatment of paediatric uncomplicated falciparum malariawith ACTs and how these ACTs affect these biochemicalindices during convalescence.This study was therefore, conducted, to compare and evaluatethe changes in biochemical indices in children pre and post treated with artemether-lumefantrine (AL) and artesunate-amodiaquine (AA) for uncomplicated Plasmodium falciparum malaria.

\section{Methods}

Data on age, sex, weight, length/height, presenting symptoms, parasitaemia and biochemical parameters (alanine transaminase (ALT), aspartate transaminase (AST), creatinine and total serum bilirubin (estimated using Cobas C111 auto-analyzer)on 114 children aged 6 months - 60 months treated for uncomplicated Plasmodium falciparum malaria were extracted from drug therapeutic efficacy (DTET), safety and tolerability study. Inclusion criteria were: history of fever in the last $24 \mathrm{hrs}$ and /or measured axillary temperature $\geq 37.5^{\circ} \mathrm{C}$, P. falciparum infection with parasitaemia $\geq 1000$ to $\leq 250,000$ parasites $/ \mu \mathrm{L}$, HIV seronegative status, and a written informed consent from parents/ guardians including readiness to comply with the follow-up visits by the parents but excluded subjects with signs of severe malaria or other common childhood diseases associated with fever for example; otitis media, tonsillitis.

Urinary tract infection was excluded by the use of combi-11 urinalysis strip test Kitts for presence of nitrite and leukocyte esterase) in a clean catch midstream urine specimen. Severe malnutrition - defined by z score $<-3 \mathrm{SD}$ or bilateral oedema. ${ }^{6}$ Patients with history of allergy to ACTs or refusal to give consent were also excluded.

HIV infection was excluded by Abbott Determine HIV-1 and 2 Whole Blood Assay Kit (Abbott Laboratory, USA). Those who where positive were to be confirmed by Western Blotting principle using Qualicode ${ }^{\mathrm{TM}} \mathrm{HIV}-1$ and 2 Kitts.

Parasite genotyping Blood spot on filter paper for parasite genotyping was obtained on Day 0 for all subjects then on any subsequent day patient was found to have parasitaemia. Polymorphic markers, Merozoites Surface Protein-1(MSP-1), MSP-2 and Glutamate-Rich Protein (GLURP) in P. falciparum isolates were used to examine the complexity of parasite populations and discriminate between recrudescent and re-infections in the study participants.

Antimalarial chemotherapy Enrollees who met the inclusion criteria were randomized into the two treatment arms to receive weight-dependent study medications artemether-lumefantrine $(20 / 120 \mathrm{mg})$ or a 3 day course of artesunate-amodiaquine $(25 \mathrm{mg} / 67.5 \mathrm{mg}$ or $50 \mathrm{mg} / 135 \mathrm{mg}$ ) according to the manufacturer's instructions. Clinical, parasitological and biochemical evaluations were carried out on Days 0,7 and 14 .

Analysis was restricted to 111 children who completed the study at day 28 (D28) follow-up visit. The number of patients treated with artemether-lumefantrine (AL) was 56 vs55 in the artesunate-amodiaquine(AA) group. Parasite densities $(\mu \mathrm{L})$ were computed using the actual baseline leukocyte counts at enrollment (D0).

Site of the study This study was conducted in General hospital Barki-Ladi, Plateau State,located $53 \mathrm{~km}$ south of Jos, the state capitalwith a population of 175, 267 (2006 National Census).

Ethical consideration Ethical/approval was received by the Institutional Health Research Ethic Committee (IHREC) of the Jos University Teaching Hospital, Jos Nigeria.

Time of the study The study was conducted between $27^{\text {th }}$ September and $16^{\text {th }}$ December, 2010.

Data management All patients' specific details and laboratory parameters were recorded in a case record form (CRF). The information was then entered into the computer using SPSS software versions 15.0 and 17.0. All patients' codes were double entered serially to avoid transcriptional errors and were only analyzed at the end of the study. Frequency tables and percentages were used to determine the means of all parameters used. Means were used to get the difference in averages between the two drugs ( $\mathrm{AL}$ and $\mathrm{AA}$ ) according to all parameter used. Student's t-test was used to calculate the significant difference between the two drugs. Standard deviation and standard error were also calculated. Levene's test for equality of variances was used to get the F and P- values. All tests of significance were two-tailed. P-value $<0.05$ was taken to indicate significant difference.

\section{Baseline characteristics of study patients}

\section{Results}

Of 649 subjects screened $282(43.5 \%)$ were febrile (temperature $\geq 37.5^{\circ} \mathrm{C}$ ). Two hundred and fifty-two (38.8\%) had positive peripheral blood film for P falciparummono-infection while 397 (61.2\%) children had negative peripheral blood film, There was no mixed infection. Of the 252 patients with parasitaemia, 114 (45.2 $\%)$ fulfilled the enrollment criteria and were randomized into one of two treatment arms to receive either artemether-lumefantrine (AL)orartesunate-amodiaquine (AA). In each treatment arm, there were 57 participants. Of the 114 enrollees, 111 (97.4\%) completed the study (Figure 1). One subject was lost to follow-up because he 
relocated from the study area to his home state for reasons not connected to the study and was followed-up for safety using mobile telephone. One subject was excluded on account of the development of signs of severe malaria and one subject was excluded from analysis owing to missing packed cell volume and parasite count data.All these three patients were excluded from data analysis. The subject with severe malaria was successfully treated with rate-controlled intravenous infusion of quinine. The study profile of all participants is as shown in Figure 1 below:

Age and sex DistributionThe mean age of study population in the AL and AA arms were 38.9 \pm 16.90 and $37.7 \pm 16.76$ respectively, $(\mathrm{p}=0.72)$. Thirty one $(55.4 \%)$ and $25(44.6 \%)$ were males and females in the AL study arm respectively while $32(58.2 \%)$ and $23(41.8 \%)$ were males and females respectively in the AA study arm, $(\mathrm{p}=0.77)$.

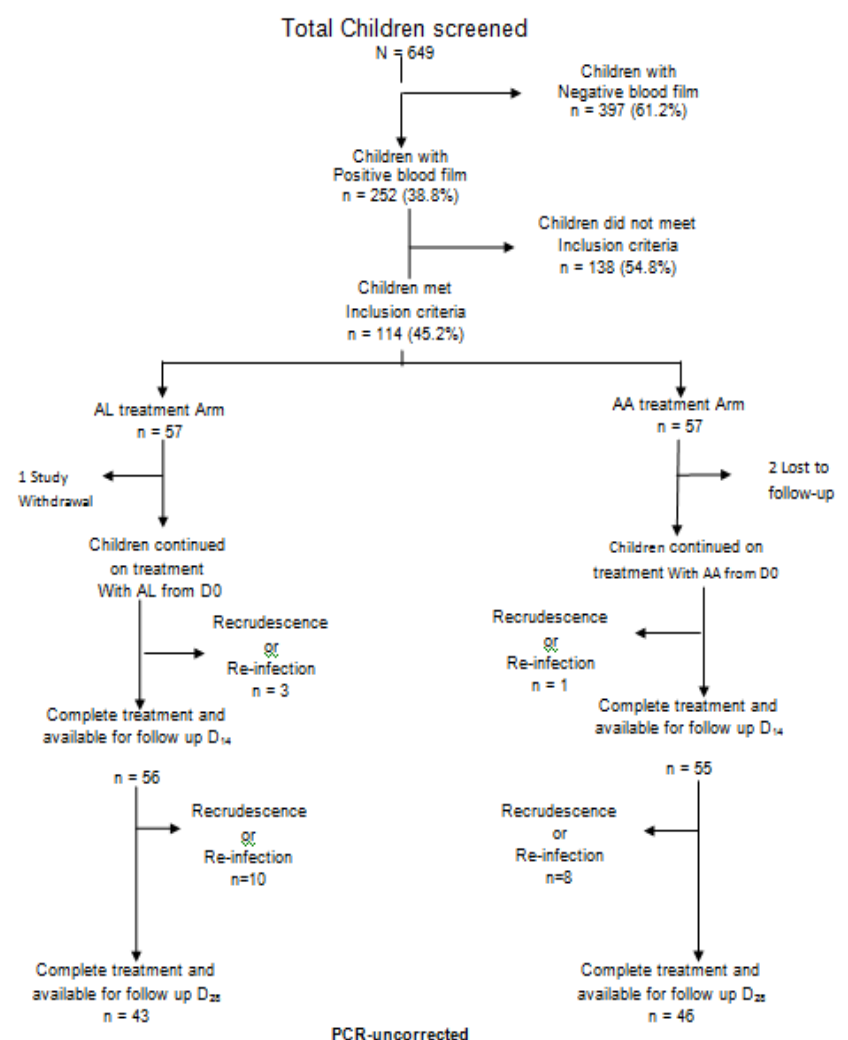

Figure 1: The profile of study subjects.

The baseline presenting symptoms were compared with the study medications in the two treatment arms; there was no significant difference, Table 1.

Table 1: Baseline Presenting Symptoms of Patients studied.

\begin{tabular}{|c|c|c|c|c|c|}
\hline Baseline symptoms & $\begin{array}{l}\text { All subjects } \\
\mathrm{N}=111(\%)\end{array}$ & $\begin{array}{l}\text { Subjects on } \mathrm{AL} \\
\text { treatment arm } \\
\mathrm{n}=56(\%)\end{array}$ & $\begin{array}{l}\text { Subjects on AA treatment } \\
\text { arm } \\
n=55(\%)\end{array}$ & $\overline{X^{2}}$ & $P$ - value \\
\hline Abdominal pain & $27(24.3)$ & $17(30.4)$ & $10(18.2)$ & 2.235 & 0.135 \\
\hline Vomiting & $23(20.7)$ & $10(17.9)$ & $13(23.6)$ & 0.564 & 0.453 \\
\hline Cough & $22(19.8)$ & $12(21.4)$ & $10(18.2)$ & 0.841 & 0.668 \\
\hline Diarrhoea & $18(16.2)$ & $9(16.4)$ & $9(16.4)$ & 0.002 & 0.967 \\
\hline Headaches & $9(8.1)$ & $6(10.7)$ & $3(5.5)$ & 1.030 & 0.310 \\
\hline Poor appetite & $4(3.6)$ & $2(3.6)$ & $2(3.6)$ & 0.000 & 0.985 \\
\hline $\begin{array}{l}\text { Mean axillary Temp } \\
\left({ }^{\circ} \mathrm{C}\right)\end{array}$ & $37.8 \pm 0.14$ & $37.8 \pm 0.98$ & $37.9 \pm 1.12$ & 0.865 & 0.684 \\
\hline$<37.5^{\circ} \mathrm{C}$ & $31(27.9)$ & $16(28.6)$ & $15(27.3)$ & & \\
\hline$\geq 37.5^{\circ} \mathrm{C}$ & $80(72.1)$ & $39(69.8)$ & $41(74.5)$ & & \\
\hline Hepatomegaly & $14(12.6)$ & $8(57.1)$ & $8(57.1)$ & 0.370 & 0.543 \\
\hline Splenomegaly & $20(18.0)$ & $10(50.50)$ & $10(50.50)$ & 0.002 & 0.965 \\
\hline Spleen Rate (\%) & $20(18.0)$ & $9(50.0)$ & $11(50.0)$ & 0.002 & 0.660 \\
\hline
\end{tabular}

$*$ Temp $=$ Temperature, $\mathrm{AL}=$ Artemether-lumefantrine, $\mathrm{AA}=$ Artesunate-lumefantrine, Pre-enrollment parasite count (Day 0) 
Pre-enrollment parasite count (Day 0) When subjects were grouped according to range-specific parasitaemia and according to study arms (AL versus AA), there was no statistical difference in the range of parasitaemia and the number of study participants in different ranges, Table 2

Table 2: Day 0 Parasite Counts of Patients studied

\begin{tabular}{|c|c|c|c|c|c|c|}
\hline $\begin{array}{l}\text { Laboratory } \\
\text { Feature }\end{array}$ & $\begin{array}{l}\text { All subjects } \\
\mathrm{N}=111\end{array}$ & $\begin{array}{l}\text { AL Treatment } \\
\operatorname{arm~n=56(\% )}\end{array}$ & $\begin{array}{l}\text { AA Treatment } \\
\operatorname{arm} \mathbf{n}=\mathbf{5 5}(\%)\end{array}$ & P-value & & \\
\hline $\begin{array}{l}\text { Mean Parasite } \\
\text { Density at } D 0(\mu 1)\end{array}$ & & $27,884.2 \pm 23,797.0$ & $27.697 .6 \pm 26,025.6$ & $27,884.2 \pm 23,797.0$ & 0.49 & 0.92 \\
\hline $1000-<5000$ & & $13(11.7)$ & $7(6.3)$ & $6(5.4)$ & 0.07 & 0.79 \\
\hline $5000-<50000$ & & $84(75.7)$ & $42(37.8)$ & $42(37.8)$ & 0.03 & 0.87 \\
\hline $50000-<100000$ & & $11(9.9)$ & $5(4.5)$ & $6(5.4)$ & 0.12 & 0.73 \\
\hline $100000-<20000$ & & $3(2.7)$ & $2(1.8)$ & $1(0.9)$ & 0.32 & 0.57 \\
\hline$>200000$ & & $0(0.0)$ & $0(0.0)$ & $0(0.0)$ & & \\
\hline
\end{tabular}

${ }^{*} \mathrm{AL}=$ Artemether-lumefantrine, $\mathrm{AA}=$ Artesunate-amodiaquine,$\mu \mathrm{l}=$ microliter

\section{Polymerase chain reaction (PCR) results}

Matched sample pairs collected before and after treatment from patients who were successfully analyzed at all the three loci MSP-1, MSP-2 and GLURP. Genotyping of these samples showed that the allelic families of MSP-1, MSP-2 and GLURP were often represented in parasite DNA derived from a single patient, indicating a polyclonal infection. Only P falciparum malaria mono-infection was identified.

Table 3: Biochemical Characteristics of Subjects studied on D0, D7 and D14

\begin{tabular}{|c|c|c|c|c|c|c|c|c|}
\hline \multirow[t]{2}{*}{ Parameter D0 } & \multicolumn{3}{|c|}{ D7 } & \multicolumn{3}{|c|}{ t-test } & \multirow[t]{2}{*}{ p-value } & \\
\hline & $\mathrm{AL}$ & AA & $\mathrm{AL}$ & AA & $\mathrm{AL}$ & AA & & \\
\hline $\operatorname{ALT}(\mathrm{IU} / \mathrm{L})$ & $20.8 \pm 18.7$ & $24.1 \pm 26.9$ & $29.7 \pm 19.5$ & $35.3 \pm 23.6$ & $27.3 \pm 12.6$ & $26.5 \pm 19.5$ & 1.921 & 0.057 \\
\hline $\operatorname{AST}(\mathrm{IU} / \mathrm{L})$ & $26.5 \pm 24.4$ & $27.4 \pm 26.7$ & $33.7 \pm 21.6$ & $34.2 \pm 21.6$ & $28.0 \pm 17.8$ & $33.0 \pm 19.2$ & 0.764 & 0.447 \\
\hline Total bilirubin & $0.8 \pm 0.9$ & $0.8 \pm 0.9$ & $0.4 \pm 0.4$ & $0.4 \pm 0.5$ & $0.4 \pm 0.6$ & $0.4 \pm 0.7$ & -0.083 & 0.934 \\
\hline$(\mathrm{mg} / \mathrm{dl})$ & & & & & & & & \\
\hline Creatinine & $36.8 \pm 35.9$ & $36.2 \pm 31.8$ & $49.6 \pm 40.7$ & $44.9 \pm 38.8$ & $35.2 \pm 27.9$ & $36.9 \pm 32.6$ & 0.287 & 0.775 \\
\hline
\end{tabular}

$* \mu m o l / d l=$ micromol per deciliter, $I U / L=$ international unit per liter, $m g /$ dl $=$ milligram per deciliter. $A L=$ Artemether-lumefantrine, $A A=$ Artesunate-amodiaquine, $D=$ day

\section{Discussion}

At enrolment, renal impairment was noticed in both treatment arms. Functional renal impairment in the form of transient decrease in endogenous clearance of creatinine was reflected in this study by the mild elevation of serum creatinine levels. ${ }^{5}$ This decrease was not significant. Serum creatinine levels normalized in both treatment arms within the first week following initiation of antimalarial chemotherapy. The finding in this study is consistent with the findings of other authors in Ogume Delta State, Nigeria. ${ }^{5,6}$ The precise mechanisms of mild renal impairment in P. falciparum malaria are multifactorial. ${ }^{6}$

No records of clinical hyperbilirubinaemiawere noticed in the study participants in AL and AA treatment arms during the study. However, at enrollment, mild and transient increases in serum bilirubin were noticedin both treatment arms. The serum bilirubin levels fell to the nadir by the D7 then rose gradually to the patients' normal values by the D14 following antimalarial chemotherapy and clearance of malaria parasites in the peripheral blood. ${ }^{8}$ These changes however, did not reach statistically significant levels. The etiopathogenesis of hyperbilirunaemia in acute malaria is multi-factorial: intravascular haemolysis of both parasitized and unparasitized erythrocytes. ${ }^{8}$ These acute but transient alterations are thought more as acute phase reactants. ${ }^{9}$

Transient disturbances in hepatic transaminases (ALT and AST) were noted during the study. Alterations in ALT and AST levels appeared comparable at enrollment in both treatment arms (AL and AA). Hepatic transaminitis were remarkable at D7follow-up visits in AL and AA treatment arms. The transaminases 
levels normalized at D14. No study participants had values greater than mean $\pm 2 \mathrm{SD}$ in both treatment arms. The reasons for these biochemical disturbances are not completely clear. They are thought tobe frequent accompaniment of acute uncomplicated P. falciparum malaria. ${ }^{9}$ The rapid normalization of these hepatic enzymes within the second week following treatment and parasite clearance wereconsidered more as acute phase response. The findings in this study agree with those found elsewhere, who for many years worked on the fields of malaria. ${ }^{9,10}$

\section{Acknowledgements}

We are sincerely grateful to the Federal Ministry of Health, (FMOH), Nigeria, for the part sponsorship of the study.

The authors have no conflicts of interest to disclose.

\section{Disclosures}

\section{References}

[1]. Bidaki ZM, Dalimi AH: Biochemical and haematological alteration in vivax malaria in Kahnouj City. J Rafsanjani Univ Med Sci1994; 3(1): 17-24

[2]. Adeosum OG, Oduola T, Akanji BO et al; Biochemical alteration in Nigerian children with acute falciparum malaria. Afri $\mathrm{J}$ Biotechnol 2007; 6 (7); 881-885.

[3]. Udosen EO: Malaria treatment using oral Melkafin; Changes in biochemical and haematological parameters in Nigerian children with uncomplicated falciparum malaria; Orient J Med 15(12)

[4]. Rajpurkar MM: Renal involvement in malaria. J Postgrad Med 40: 134-4

[5]. Ahmed SH, Danish T, Faridi MMA, Ahmen AJ, Fakhir S and Khan AS. Renal Function in Acute malaria in Children. J. Trop Pediatr (1989) 35(6) 291-294. http://tropej.oxfordjournals.org/content/35/6/291.

[6]. Rapheal C E and Gideon I O. Assessment of Renal Function of Nigerian Children infected with Pl. falciparum. Int. J of Med and Med Scie, 2010, 2(9) 251-215.

[7]. Stephen E and Christian DM: Artemether-lumefantrine in the treatment of Uncomplicated Plasmodium falciparum malaria. Dove Med J, Ther and Clin Risk Mgt, 2009, 5: 805-815.

[8]. Mishra SK, Mohapatra S, Mohanty SY; Jaundice in falciparum malaria, JIACM, 4(1):12-3

[9]. Singh R, Kaur M and Arora D. A Prospective Study of Hepatic Involvement In P. falciparum malaria. J Clin and Diag Res, [serial online], 2010, 4: 2190-2197.

[10]. Mishra SK, Mohanty S, Das BS, Patnaik JK, Satpathy SK, Mohanty D et al. Hepatic Changes in Pl. falciparum malaria, NCBI Research.Indian J Malariol. 1992, 29(3):167-71. 